\title{
Steady state and picosecond time-resolved photophysics of a benzimidazolocarbocyanine dye
}

\author{
Serdar Özçelik* \\ Chemistry Department, Bilkent University, Bilkent-06533 Ankara, Turkey
}

Received 7 August 2000; received in revised form 11 July 2001; accepted 15 August 2001

\begin{abstract}
We report the steady-state and the excited state properties of $1,3,1^{\prime}, 3^{\prime}$-tetraethyl-5, 6, 5' $6^{\prime}$-tetrachlorobenzimidazolocarbocyanine iodide (TTBC) in various solvents. The magnitude of the Stokes shift and the average transition energy proves that the structure of the fluorescent state should be very similar to that of the ground-state structure. The fluorescence lifetimes and quantum yields indicate that non-radiative processes are collectively much more effective than the radiative processes. It is therefore suggested that the non-radiative processes, which are driven by the medium around the TTBC molecule control the excited state dynamics. (c) 2002 Elsevier Science B.V. All rights reserved.
\end{abstract}

Keywords: Photophysics; Cyanine dyes; Multi-frequency phase-modulation method; Picosecond fluorescence lifetimes

\section{Introduction}

The photophysics and photochemistry of cyanine dyes have been studied extensively [1-10]. It has been demonstrated that photoisomerisation and internal conversion, in general, participate in the deactivation of the excited state, and the restriction of these processes enhances fluorescence efficiency of cyanine dyes.

Cyanine dyes have found utility as spectral sensitizers for silver halide microcrystals in photoimaging industry [11], as mode-locking dyes and saturable absorbers for ultrafast lasers [12]. Cyanine dyes are promising materials for a wide variety of applications from photodynamic therapy to non-linear optics [13-21].

\footnotetext{
*Tel: +90-312-290-2153; fax: +90-312-266-4579.

E-mail address: sozcelik@fen.bilkent.edu.tr (S. Özçelik).
}

Superradiance (mirrorless laser-like emission) from aggregated benzimidazolocarbocyanines [2224] and selective binding to mitochondria [15-17] stimulate the investigation of the photophysical properties of $1,3,1^{\prime}, 3^{\prime}$-tetraethyl-5,6,5', $6^{\prime}$-tetrachlorobenzimidazolocarbocyanine iodide (TTBC, see inset in Fig. 1 for the structure). Basic photophysical properties are important as a reference for understanding the aggregation behavior. Although, there exist extensive literature for photophysics and photochemistry of cyanine dyes, a detailed investigation of the excited state properties of TTBC in various solvents was not carried out.

The phase-modulation fluorescence spectroscopy is especially convenient to measure excitedstate properties because the light source, a cw xenon light, of a phase-modulation fluorescence spectrophotometer has a greater flexibility to provide a tunable selective excitation wavelength. 

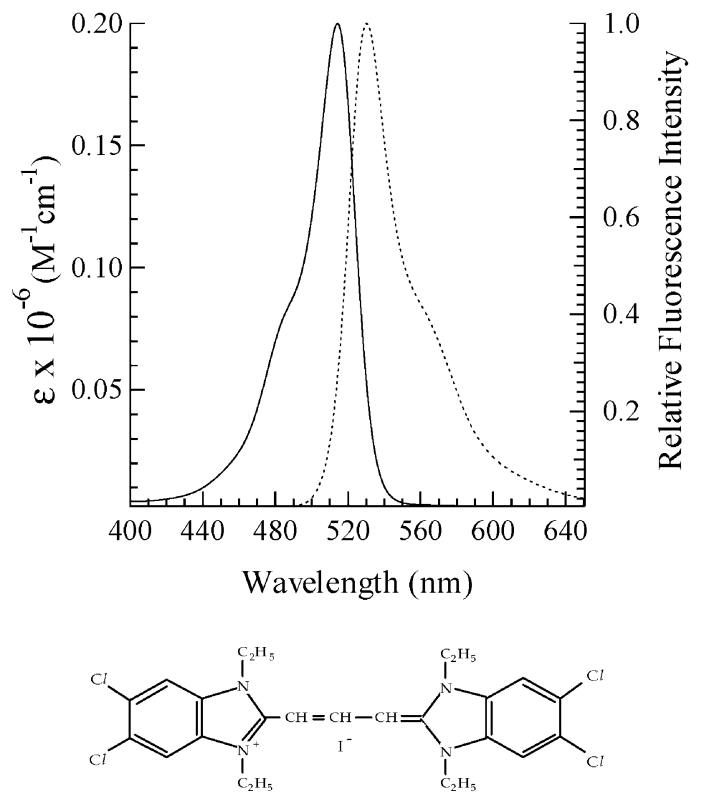

Fig. 1. Absorption and fluorescence spectra of TTBC in methanol at room temperature. Chemical structure of TTBC is inserted. $[\mathrm{TTBC}]=10 \mu \mathrm{M}$ for absorption.

The acquisition time is relatively fast, and low excitation power eliminates multiphoton effects. The multi-frequency measurements ensure to analyze the multi-exponential and the non-exponential decays arising from complex phenomenon [25,26]. Fluorescence lifetimes measured by three different methods, i.e. time correlated single photon counting, phase-modulation and streak camera, gave the same results for the aggregated systems [26].

The purpose of the present study is to characterize the steady-state and excited-state properties of TTBC molecules in various solvents, and to understand how solvent affects the optical dynamics.

The experimental results of the steady-state and the time-resolved fluorescence/phosphorescence spectroscopies of TTBC in various solvents are presented in this paper. In addition, the related photophysical parameters are also reported.

\section{Experimental}

TTBC was purchased from Accurate Chemical and Scientific Co. (Westbury, New York). The chemical purity of TTBC was checked by a Shimadzu HPLC and found to be $99.90 \%$ pure. The spectroscopic grade solvents and LUDOX colloidal silica sol used in this study were purchased from Aldrich Chemical Co. (Milwaukee, WI). All chemicals were used without further purification.

The absorption spectra were recorded using a Perkin-Elmer Lambda-19 UV-vis-NIR spectrophotometer. The steady-state fluorescence excitation and emission, and synchronised luminescence spectra were acquired using a SPEX Fluorolog- $\tau 2$ fluorometer. The synchronized luminescence is obtained by scanning excitation and emission monochromators simultaneously [27]. The SPEX phosphorimeter module for phosphorescence and triplet decay time measurements was utilized with acquisition parameters: flash duration of $3 \mu \mathrm{s}, 150$ flashes for each spectral point and 20 flashes/s. For triplet decay measurements, the data were collected up to $50 \mu \mathrm{s}$.

The fluorescence quantum yields were determined relative to rhodamine $6 \mathrm{G}$ in ethanol $(\Phi=$ 0.95 ) as the reference. We compared the spectrally corrected and integrated emission intensity of the sample to that of the reference. The dilute reference and the samples were prepared at $5.0 \mu \mathrm{M}$ concentrations. Right angle geometry and a sample cell with $0.15 \mathrm{~cm}$ path length in the direction of both excitation and emission were used for the measurements. The corrections required for wavelength response of the emission monochromator-photomultiplier combination were made with correction factors supplied by the vendor.

The fluorescence lifetime measurements were acquired by SPEX Fluorolog- $\tau 2$ fluorometer; this instrument uses the multi-frequency phasemodulation technique [25] in the range from 0.5 to $330 \mathrm{MHz}$. For fluorescence lifetime measurements, a cw xenon light source is directed to a Pockels cell for the modulation. Approximately $8 \%$ of modulated excitation light from the Pockels cell is directed to the reference detector and the remainder to the sample. A reference standard, a light scatterer such as LUDOX colloidal silica sol, is required to measure the fluorescence lifetimes. The fluorescence lifetime determination was made 
through an analysis, which utilises the relative phase shift, and relative demodulation of the sample compared to that of the reference standard [25]. The analysis and nonlinear least-squares fitting (Marquardt-Levenson minimization algorithm) were performed with Globals Unlimited (Urbana, IL) analysis software.

\section{Results and discussion}

Fig. 1 shows the absorption spectrum of TTBC that consists of a band at ca. $514 \mathrm{~nm}$ with a shoulder at ca. $480 \mathrm{~nm}$; assigned, respectively, to the $0-0$ (bandwidth ca. $791 \mathrm{~cm}^{-1}$ ) and $1-0$ (bandwidth ca. $1600 \mathrm{~cm}^{-1}$ ) vibronic transitions. The extinction coefficient is calculated to be $2.0 \times 10^{5} \mathrm{M}^{-1} \mathrm{~cm}^{-1}$, which is in agreement with the literature [28]. The large value of the extinction coefficient indicates an extensive conjugation of $\pi$ electrons suggesting a planar structure. However, the X-ray structure analysis of the TTBC single crystals indicates that the molecule is approximately planar despite extensive conjugation of $\pi$ electrons, and that the polymethine chain is twisted about $4^{\circ}$ to minimize steric strain [29]. In the single crystal form, the cationic TTBC molecules pack plane to plane and end to end in sheets. It was also provided that the edge of the molecular plane is $2.08 \mathrm{~nm}$, and that the projected area is $0.738 \mathrm{~nm}^{2}$. Moreover, it was demonstrated that the ethyl substituents attached to nitrogen atoms in imidazoly rings protrude to the molecular plane.

Fig. 1 also shows the fluorescence spectrum of TTBC in methanol, excited at ca. $450 \mathrm{~nm}$. The fluorescence has maximum at ca. $528 \mathrm{~nm}$, and a shoulder at ca. $560 \mathrm{~nm}$. It was found that the fluorescence maximum did not shift as a function of the excitation wavelength. The synchronised luminescence spectrum exhibits a single peak. This single peak indicates that there is only one fluorescent excited-state. This fluorescent state, as suggested by the overlap of the synchronised luminescence peak and the fluorescence excitation spectrum, should be the first excited singlet state.

The steady-state spectra of TTBC in various solvents are also acquired. The measured photophysical parameters are summarized in Table 1. Some solvent effects (solvatochromism) on the photophysics are observed. Fig. 2 shows a plot of absorption and fluorescence transition energies as a function of the orientational polarizability of the solvent, $\Delta f=\left[(\varepsilon-1 / 2 \varepsilon+1)-\left(n^{2}-1 / 2 n^{2}+1\right)\right]$, where $\varepsilon$ is the dielectric constant and $n^{2}$ is the refractive index of the solvent [25]. It is shown that the transition energies are blue-shifted with increasing orientational polarizability.

Table 1

The photophysical parameters of TTBC at room temperature

\begin{tabular}{llllllllll}
\hline Parameters & $\mathrm{MeOH}$ & $\mathrm{EtOH}$ & $\mathrm{PrOH}$ & $\mathrm{BuOH}$ & $\mathrm{HexOH}$ & $\begin{array}{l}\text { Ethylene } \\
\text { glycol }\end{array}$ & Glycerol $^{\mathrm{a}}$ & Acetonitrile & DMSO \\
\hline$\lambda_{\text {abs }}(\mathrm{nm})^{\mathrm{b}}$ & 514 & 516 & 518 & 520 & 521 & 518 & 521 & 515 & 522 \\
$\lambda_{\text {fluo }}(\mathrm{nm})^{\mathrm{c}}$ & 528 & 530 & 532 & 534 & 535 & 532 & 535 & 529 & 536 \\
$\Delta v_{\text {Stokes }}\left(\mathrm{cm}^{-1}\right)$ & 515 & 512 & 508 & 504 & 502 & 508 & 502 & 513 & 500 \\
$\lambda_{\text {fluo }}{ }$ & 0.016 & 0.023 & 0.033 & 0.042 & 0.058 & 0.075 & 0.360 & 0.024 & 0.055 \\
$\tau_{\text {fluo }}(\mathrm{ps})$ & $47 \pm 5$ & $69 \pm 5$ & $97 \pm 10$ & $127 \pm 10$ & $168 \pm 10$ & $220 \pm 15$ & $996 \pm 15$ & $65 \pm 5$ & $153 \pm 10$ \\
$\chi^{2}$ & 0.73 & 1.20 & 0.98 & 1.29 & 1.55 & 1.12 & 0.81 & 1.44 & 1.19 \\
$k_{\mathrm{r}} \times 10^{9}\left(\mathrm{~s}^{-1}\right)$ & $0.34 \pm 0.08$ & $0.33 \pm 0.05$ & $0.34 \pm 0.05$ & $0.33 \pm 0.04$ & $0.34 \pm 0.02$ & $0.34 \pm 0.03$ & $0.36 \pm 0.02$ & $0.37 \pm 0.06$ & $0.36 \pm 0.04$ \\
$k_{\mathrm{nr}} \times 10^{9}\left(\mathrm{~s}^{-1}\right)$ & $20.9 \pm 4.7$ & $14.1 \pm 1.7$ & $9.96 \pm 1.63$ & $7.54 \pm 0.95$ & $5.61 \pm 0.35$ & $4.20 \pm 0.35$ & $0.64 \pm 0.01$ & $15.0 \pm 2.43$ & $6.17 \pm 0.62$ \\
$k_{\mathrm{nr}} / k_{\mathrm{r}}$ & 62 & 43 & 30 & 23 & 17 & 12 & 2 & 41 & 17 \\
\hline
\end{tabular}

${ }^{\text {a }}$ For gylcerol, a double exponential decay: the shortest lifetime 211 ps with $4 \%$ of the emission and 996 ns with $96 \%$ of the emission.

${ }^{\mathrm{b}}$ The maximum of absorption spectrum.

${ }^{\mathrm{c}}$ The maximum of fluorescence spectrum.

${ }^{\mathrm{d}}$ Absorbance values were less than 0.06 . Uncertainity in measurements is equal to \pm 0.002 for all samples and reference. 


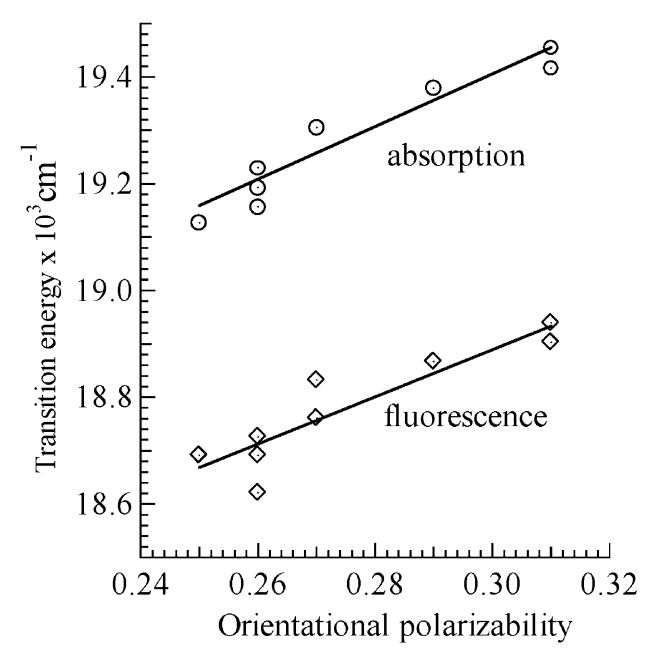

Fig. 2. Absorption and the fluorescence transition energies as a function of orientational polarizability. The lines are used to guide the eye.

The average transition energy is defined [30] as follows:

$\frac{\left(\bar{v}_{\mathrm{abs}}+\bar{v}_{\mathrm{emis}}\right)}{2}=\Delta G_{\mathrm{V}}^{0}+\omega_{\mathrm{E}}(\varepsilon)-\omega_{\mathrm{G}}(\varepsilon)$.

where $\Delta G_{\mathrm{V}}^{0}$ is the change in Gibbs energy by electronic excitation in vacuum, and should be constant. $w_{\mathrm{G}}(\varepsilon)$ and $w_{\mathrm{E}}(\varepsilon)$ are work functions for a molecule (in its ground and excited states, respectively) to be transfered from vacuum into a solvent with a dielectric constant $\varepsilon$. The average transition energy is around $19,000 \mathrm{~cm}^{-1}$ and remains almost unchanged as shown in Fig. 3. The negligible variation, $250 \mathrm{~cm}^{-1}$ corresponding to a $3 \%$ change from the average, implies that the charge distribution in the ground- and fluorescentstate would be approximately equal to each other regarding the orientational polarizability of solvents. Therefore, it is proposed that the electronic structure of the fluorescent state with respect to the ground state should be similar.

The additional experimental evidence for this proposal arises from the magnitude of Stokes shift in various solvents. The sensitivity of Stokes shift to orientational polarizability reflects the change in dipole moment occurring upon excitation and subsequent orientation of the solvent molecules.

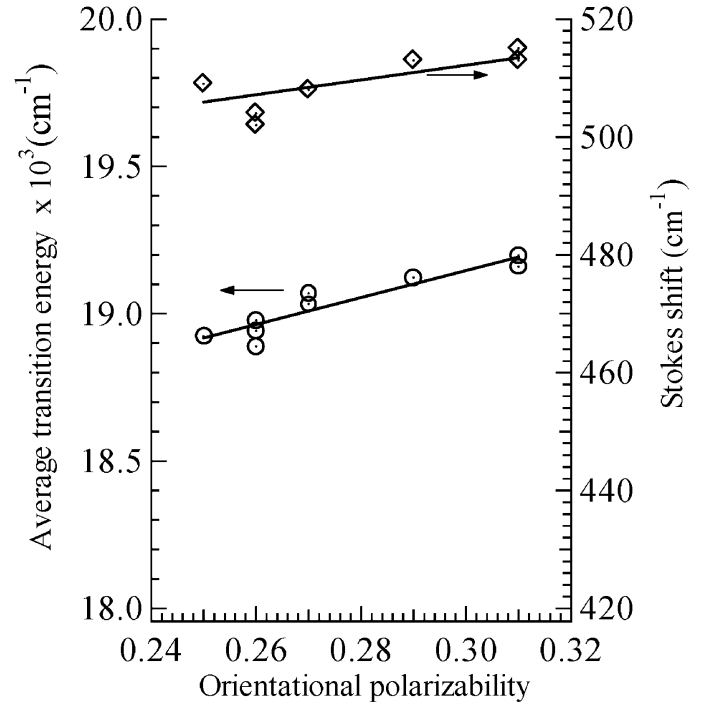

Fig. 3. Average transition energy (the left axis) and Stokes shifts (the right axis) as a function of orientational polarizability that is defined in the text. The lines are used to guide the eye.

Fig. 3 shows the variation of Stokes shift with orientational polarizability of the solvents. The magnitude of Stokes shift is about $510 \mathrm{~cm}^{-1}$ and does not vary with the orientational polarizability. A typical magnitude of Stokes shift in some cyanines due to a change in dipole moments varies in the range of $2500-5000 \mathrm{~cm}^{-1}$ [30-34]. The Lippert equation $[25,30]$ expresses the Stokes shift in terms of the intramolecular reorganization energy, $\lambda_{\mathrm{IN}}$; the orientational polarizability, $\Delta f$; the difference in dipole moments of the groundand excited-states, $\left(\mu_{\mathrm{E}}-\mu_{\mathrm{G}}\right)$; and the characteristic dimension, $a$, of the molecule under investigation.

$$
\Delta \bar{v} \equiv \bar{v}_{\mathrm{abs}}-\bar{v}_{\mathrm{emis}}=2 \frac{\lambda_{\mathrm{IN}}}{h c}+\frac{\left(\vec{\mu}_{\mathrm{E}}-\vec{\mu}_{\mathrm{G}}\right)^{2}}{2 \pi \varepsilon_{0} h c a^{3}} \Delta f .
$$

Eq. (2) predicts that the Stokes shift should linearly increase with the orientational polarizability. This, however, does not agree with the experimental results we present. To satisfy the experimental results, the second term in Eq. (2) should be zero or negligible with increasing orientational polarizability. This infers that the magnitude of the dipole moments in the ground and fluorescent state should be very similar. 
Therefore, the first term expressing the intramolecular reorganization energy should be responsible for the observed Stokes shift. We estimate that the intramolecular reorganization energy for TTBC at room temperature is about $250 \mathrm{~cm}^{-1}$. The medium around TTBC molecule could easily induce the non-radiative transitions because of the very low activation energy for the intramolecular reorganization. Therefore, very fast non-radiative transitions are expected. In fact, this is what we observe as discussed in the following part.

The phase delays $(\phi)$ and demodulation factors ( $m$ ) are measured to determine the apparent phase and modulation lifetimes, $\tau_{\mathrm{p}}$ and $\tau_{\mathrm{m}}$, respectively [25],

$\tau_{\mathrm{p}}=\omega^{-1} \tan \phi$,

$\tau_{\mathrm{m}}=\omega^{-1}\left(\frac{1}{m^{2}-1}\right)^{1 / 2}$

where $\omega$ is the modulation frequency. Fig. 4 shows the representative plots of the phase-modulation measurements in methanol and glycerol. The fluorescence lifetimes of the TTBC in methanol and glycerol at room temperature are calculated to be $47\left(\chi^{2}=0.73\right)$ and $996 \mathrm{ps}\left(\chi^{2}=0.81\right)$, respectively. Fig. 4 is a clear representation of a wide range (from $47 \mathrm{ps}$ to $1.0 \mathrm{~ns}$ ) and accurate lifetime measurements with the multi-frequency phasemodulation method. The fluorescence lifetimes in other solvents are summarized in Table 1. The fluorescence lifetime clearly manifests solvent dependency. The nature of this dependency will be discussed in combination with the quantum yield measurements.

The fluorescence quantum yields were obtained as explained in the experimental part and the results are summarized in Table 1. The fluorescence quantum yields are also sensitive to the solvent properties. The quantum yield is decreased in a more polar environment such as methanol and increased in a highly viscous solvent such as glycerol that prevents easy rotations and vibrations of TTBC. On the other hand, the less viscous and highly polar solvents provide TTBC a freedom to rotate and vibrate which can be the possible sources of the non-radiative transitions. These

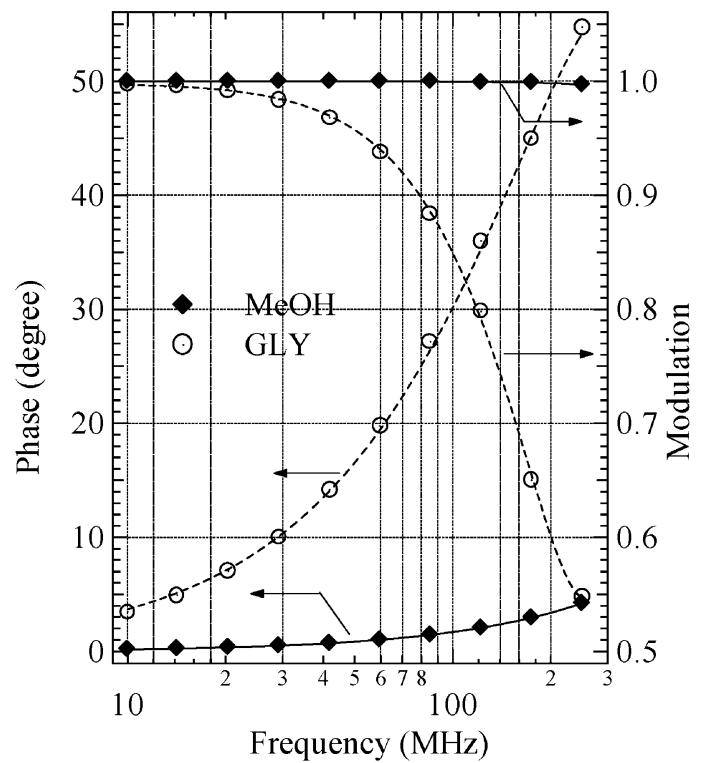

Fig. 4. Representative phase-modulation plots. Excitation wavelength was $480 \mathrm{~nm}$ for methanol and glycerol. Emission wavelengths were set to 528 and $535 \mathrm{~nm}$, respectively for methanol and glycerol. A reference was a dilute colloidal silica solution. The lines represent the best fits to the data.

observations are in concert with the other cyanine dyes [1-10].

The fluorescence lifetime and the quantum yield are related to the radiative $\left(k_{\mathrm{r}}\right)$ and non-radiative $\left(k_{\mathrm{nr}}\right)$ rate constants by the photophysical equations $k_{\mathrm{r}}=\frac{\phi_{\mathrm{f}}}{\tau_{\mathrm{f}}}$

$k_{\mathrm{nr}}=\frac{1-\phi_{\mathrm{f}}}{\tau_{\mathrm{f}}}$.

Upon substituting the measured fluorescence lifetimes and quantum yields, it is calculated that $k_{\mathrm{r}}=(0.34 \pm 0.08) \times 10^{9} \mathrm{~s}^{-1}$ and $k_{\mathrm{nr}}=(20.9 \pm$ 4.7) $\times 10^{9} \mathrm{~s}^{-1}$ for TTBC in methanol. This calculation indicates that the non-radiative transition rate is sixty-two times faster than the radiative rate. The solvent independent radiative lifetime is calculated to be $2.95 \mathrm{~ns}$.

The phosphorescence spectrum and the triplet lifetime of TTBC in ethanol were recorded at $77 \mathrm{~K}$ by using a SPEX phosphorimeter module. No 
phosphorescence signal was detected at room temperature. At $77 \mathrm{~K}$, the phosphorescence spectrum has a maximum at ca. $552 \mathrm{~nm}$ with an excitation at $514 \mathrm{~nm}$. The triplet lifetime was measured to be $4.0 \mu$ s with a single exponential decay. The fast triplet decay time and the extremely weak phosphorescence signal suggest that the non-radiative transitions in triplet manifold should be the preferred processes. However, further studies are needed to obtain triplet state properties, which is not the scope of this paper.

Fig. 5 represents how the radiative and nonradiative rate constants vary with solvent's viscosity and orientational polarizability. The radiative rate constant is independent of the medium which, indicates that the electronic structure of the fluorescent state is insensitive to the environmental perturbation induced by the solvents. This deduction supports the proposal presented here, which was reached by the steady-state spectroscopy. The non-radiative rate constant can be represented as a

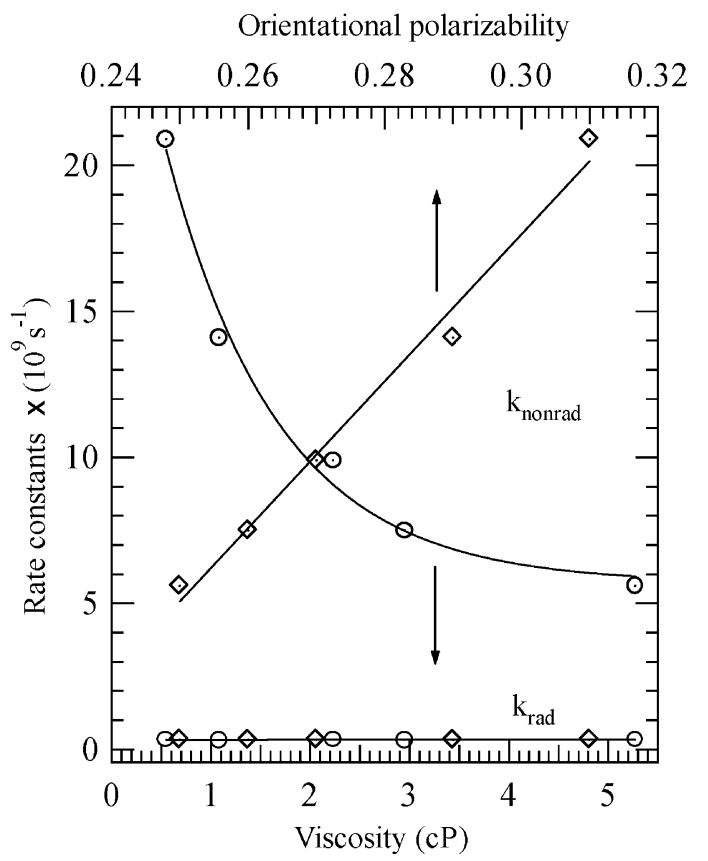

Fig. 5. Radiative and non-radiative rate constants as a function of orientational polarizability and viscosity of the solvents. The data are for primary alcohols. The lines are used to guide the eye. sum of the rates associated with internal conversion, photoisomerization, intersystem crossing and specific solvent effects. There is a consensus in the literature [1-10,35-41] on the nature of nonradiative transitions: internal conversion and photoisomerisation are the major deactivation pathways of the excited singlet state of cyanines. This consensus is based on observations such as extremely low intersystem crossing quantum yields [35-37] and as very short fluorescence lifetimes of cyanine dyes in low viscosity solvents [37-41]. The results presented in this paper provide further evidence in support of this consensus.

A closer inspection of Fig. 5 confirms that the non-radiative rate constant increases linearly with orientational polarizability while it decreases exponentially with increasing viscosity. The pronounced dominance of viscosity suggests that the photoisomerization and internal conversions are the dominant non-radiative events in the excited states of TTBC. It is well known that a change in the twisting angle around a carbon-carbon bond of the polymethine chain for cyanines causes photoisomerisation [1-10].

The ground and excited state potential energy surfaces as a function of the twisting angle around a carbon-carbon bond of the polymethine chain were computed for symmetric carbocyanines $[42,43]$. Rodriguez et al. also calculated the electric dipole moment with respect to the center of electrical charges. These calculations showed that the dipole moment slightly varies with the twisting angle; and around $90^{\circ}$ a sudden change appears in the magnitude of the dipole moments of the ground and excited states. This computation agrees with the well-known observation: a sudden increase with a sharp peak in the potential energy around $90^{\circ}$ of the twisting angles $[1,6,10]$. Ultrafast photoisomerization of symmetric cyanines has been modeled by Sanchez-Galvez et al [44]. The streptocyanines $\left(\mathrm{NH}_{2}-(\mathrm{CH})_{n}-\mathrm{NH}_{2}^{+}, \quad n=3,4,5\right)$ were used as model compounds for the calculations. A model for trimethine cyanine includes two vibrational modes: the initial totally symmetric skeletal stretching in the Franck-Condon region and the non-totally symmetric torsional motion. These modes lead to a twisted configuration in which the ground and the excited state potential 
surfaces cross at a conical intersection. It is also demonstrated that the ultrafast deactivations in the femtosecond regime is activated by a barrierless potential energy surface activates. The charge localization along the reaction coordinate is emphasised as triggering the ultrafast relaxation of the trimethine model compound. On the other hand, the model for the longer methine cyanines (penta- and hepta-methine) predicts that the stretching and torsional models are not coupled along the excited state reaction coordinate. The existence of a planar minimum along the reaction path and an activation barrier for isomerization are the predictions of the model. The existence of the planar minimum before the transition state for the longer methine cyanines is very important because it facilitates relaxations to the normal ground state by radiative and non-radiative processes. In our study, we select a spectral detection window by setting the slit widths of monochromators to $4.0 \mathrm{~mm}$ yielding to $16 \mathrm{~nm}$ of bandwidth to measure the normal form lifetime. This spectral window substantially lowers the contribution of the photoisomer fluorescence, if any. It is reported that the photoisomer emission usually shifts to longer wavelengths $[6,10]$. The observations of single exponential decays indicate that there is just one fluorescent state, that is the normal form. If there had been more than one fluorescent species, then all of them should follow the same kinetic behaviour. One exception in these series of experiments is the case of TTBC in glycerol: a double exponential decay with 211 $(4 \%)$ and $996 \mathrm{ps}(96 \%)$ indicating that the contribution of photoisomer to the observed dynamics is substantially small.

We might note the apparent differences in the results for TTBC fluorescence lifetime and quantum yield measurements performed by O'Brien et al. [37] and this study. In the former, TTBC chloride in methanol was used and a fluorescence lifetime of $550 \mathrm{ps}$ and fluorescence quantum yield of 0.1 were obtained. While in this study TTBC iodide in methanol is used and the fluorescence lifetime of $47 \mathrm{ps}$ and the fluorescence quantum yield of 0.016 were measured. However, the radiative lifetime is almost the same for both TTBC iodide and TTBC chloride. The difference between the current work and that of O'Brien et al. could be attributed to the counterion. We acquired high-performance liquid chromatography (HPLC) of the TTBC iodide to eliminate the impurity quenching and found that the sample was $99.90 \%$ pure. Tatikolov et al. reported that the interaction between a benzimidazolocarbocyanine cation and iodide counterion forms an ion-pair in low polar medium and leads to enhancement of the internal conversion rate [45]. However, the ion-pair formation in methanol is not expected. The size and effective charge density of iodide comparing to the chloride may affect the charge distribution and the orientation of solvent molecules. This would change the local field that is the effective orientational polarizability of the medium resulting in enhanced non-radiative rates. Heavy atom effects may be also considered for the discrepancy. Additional studies are necessary to clarify this discrepancy.

\section{Conclusions}

We report the steady state and excited state properties of TTBC in various solvents. The magnitudes of the Stokes shift and the average transition energy suggest that the structure of TTBC in the fluorescent-state should be very similar to that of the ground-state structure. However, TTBC in the excited-state may adopt a different conformation of a photoisomer, which is not observed for the solvents except glycerol, within the selected spectral detection window.

It is demonstrated that the quantum yields and the fluorescence lifetimes increase with solvent viscosity and decrease with solvent polarity. This indicates that the non-radiative processes are collectively much more effective than the radiative processes. We therefore conclude that the medium around the TTBC molecules controls the photophysical properties. The internal conversions and photoisomerisation are considered as the main deactivation channels for the excited state. The counterion seems also to be effective on the excited-state dynamics of TTBC.

It is desirable and of considerable importance to calculate/measure change in charge distribution 
and the dipole moments in the ground and excited states of carbocyanines to provide further confirmation.

\section{Acknowledgements}

I gratefully acknowledge Prof. Daniel L. Akins of the City College of the City University of New York, for his support. I also thank Dr. James Mattheis and Dr. Sal Atzini, Fluorescence Application Laboratory of JYHORIBA, Edison, NJ for providing technical assistance for phosphorescence measurements.

\section{References}

[1] C. Rulliere, Chem. Phys. Lett. 43 (1976) 303.

[2] A.K. Chibisov, G.V. Zakharova, H. Görner, Y.A. Sogulyaev, I.L. Mushkalo, A.I. Tolmachev, J. Phys. Chem. 99 (1995) 886.

[3] D. Noukakis, M. Van der Auweraer, S. Toppet, F.C. De Schryver, J. Phys. Chem. 99 (1995) 11860.

[4] R.E. Di Paolo, L.B. Scaffardi, R. Duchowicz, G.M. Bilmes, J. Phys. Chem. 99 (1995) 13796.

[5] M.S. Churio, K. Angermund, S.E. Braslavsky, J. Phys. Chem. 98 (1994) 1776.

[6] M.M. Award, P.K. McCarthy, G.J. Blanchard, J. Phys. Chem. 98 (1994) 1454.

[7] N. Serpone, M.R.V. Sahyun, J. Phys. Chem. 98 (1994) 734.

[8] P.F. Aramendia, R.M. Negri, E.S. Roman, J. Phys. Chem. 98 (1994) 3165.

[9] V. Khimenko, A.K. Chibisov, H. Görner, J. Phys. Chem. A 101 (1997) 7304.

[10] A. Penzkofer, M. Ramelsperger, M. Wittman, Chem. Phys. 208 (1996) 137.

[11] P.B. Gilman, Photo. Sci. Eng. 18 (1974) 418.

[12] F.P. Schäfer, in: Dye Lasers, 3rd Ed., Springer, Heidelberg, 1990.

[13] M. Krieg, R.W. Reymond, Photochem. Photobiol. 57 (1993) 472.

[14] M. Krieg, J.M. Bilitz, M.B. Srichai, R.W. Reymond, Biochim. Biophys. Acta 1199 (1994) 149.

[15] M. Reers, T.W. Smith, L.B. Chen, Biochemistry 30 (1991) 4480.

[16] S. Smiley, M. Reers, C. Mottola-Hartshorn, M. Lin, A. Chen, T.W. Smith, G.D. Steele Jr., L.B. Chen, Proc. Natl. Acad. Sci. USA 88 (1991) 3671.
[17] A. Cossarizza, C.M. Baccarani, G. Kalashnikova, C. Franchesci, Biochim. Biophys. Res. Commun. 197 (1993) 40.

[18] E. Masano, C. Adachi, T. Tsutsui, S. Saito, Thin Solid Films 210/211 (1992) 468.

[19] F. Sasaki, S. Kobayashi, Appl. Phys. Lett. 63 (1993) 2887.

[20] Y.J. Wang, Opt. Soc. Am. B 8 (1991) 981.

[21] M. Furuki, O. Wada, L.S. Pu, L.Y. Sato, H. Kawashima, T. Tani, J. Phys. Chem. B 103 (1999) 7607.

[22] S. Ozcelik, D.L. Akins, Appl. Phys. Lett. 71 (1997) 3057.

[23] S. Ozcelik, D.L. Akins, J. Phys. Chem. B 101 (1997) 3021.

[24] S. Ozcelik, I. Ozcelik, D.L. Akins, Appl. Phys. Lett. 73 (1998) 1949.

[25] J.R. Lakowicz; In Principles of Fluorescence Spectroscopy, 2nd Edition, Kluwer Academic/Plenum Publishers, New York, 1999, Chapters 5 and 6, 141-184, and $187-193$.

[26] D.L. Akins, S. Ozcelik, H.-R. Zhou, C. Gou, J. Phys. Chem. 100 (1996) 14390.

[27] T. Vo-Dinh, Appl. Spectrosc. 36 (1982) 576.

[28] B. Zuckerman, Photo. Sci. Eng. 11 (1967) 156.

[29] D.L. Smith, H. R. Luss Acta Crystallogr. Sec. B 28 (1972) 2793.

[30] P. Fromherz, J. Phys. Chem. 99 (1995) 7188.

[31] C. Rocker, A. Heilemann, P. Fromherz, J. Phys. Chem. 100 (1996) 12172.

[32] U. Narang, C.F. Zhao, J.D. Bhawalkar, F.V. Bright, P.N. Prasad, J. Phys. Chem. 100 (1996) 4521.

[33] C.F. Zhao, R. Gvishi, U. Narang, G. Ruland, P.N. Prasad, J. Phys. Chem. 100 (1996) 4526.

[34] J. Zheng, D. Wu, J. Zhai, C. Huang, W. Pei, X. Gao, Phys. Chem. Chem. Phys. 1 (1999) 2345.

[35] A.V. Buettner, J. Chem. Phys. 46 (1967) 1398.

[36] R.A. Berg, A. Ron, J. Chem. Phys. 59 (1973) 3289.

[37] D.F. O'Brien, T.M. Kelly, L.F. Costa, Photo. Sci. Eng. 18 (1974) 76.

[38] G.R. Fleming, A.E.W. Knight, J.M. Morris, R.J. Robbins, G.W. Robinson, Chem. Phys. Lett. 49 (1977) 1.

[39] D.H. Waldeck, G.R. Fleming, J. Phys. Chem. 85 (1981) 2614.

[40] S.P. Velsko, G.R. Fleming, Chem. Phys. 65 (1982) 59.

[41] S. Murphy, B. Sauerwein, H.G. Drickamer, G.B. Schuster, J. Phys. Chem. 98 (1994) 13476.

[42] J. Rodriguez, D. Scherlis, D. Estrin, P.F. Aramendia, R.M. Negri, J. Phys. Chem. B 101 (1997) 6998.

[43] J. Park, Dyes Pigments 46 (2000) 155.

[44] A. Sanchez-Galvez, P. Hunt, M.A. Robb, M. Olivucci, T. Vreven, H.B. Schlegel, J. Am. Chem. Soc. 122 (2000) 2911.

[45] A.V. Tatikolov, Kh.S. Dzhulibekov, L.A. Shvedova, V.A. Kuzmin, A.A. Ischencko, J. Phys. Chem. 99 (1995) 6525 . 